\title{
Estudios de Asia Oriental en el ámbito hispanoparlante: la apuesta de la Universidad de Málaga
}

\author{
Manuel MontalbáN* \\ María AuXiliadora DurÁN**
}

* Profesor de Antropología Social en Estudios de Asia Oriental, Universidad de Málaga.

** Profesora de Psicología Social en Estudios de Asia Oriental, Universidad de Málaga.

Coordinadora del Título de Grado de Estudios de Asia Oriental, mención Corea,

Universidad de Málaga.

\section{Resumen}

El presente artículo parte de la idea general de que el concepto clásico de educación se ve profundamente afectado por la globalización contemporánea. Una de las más importantes expresiones de la globalización en el ámbito educativo es la internacionalización, sobre todo en la etapa de la formación universitaria. Así, las universidades de todo el mundo consideran la internacionalización un elemento privilegiado en sus planes de desarrollo y futuro.

Es en este contexto que entre las universidades españolas crece el interés por intensificar la internacionalización con universidades de Asia Oriental, sobre todo China, Japón y Corea del Sur. De igual modo, se ha ampliado y diversificado en los últimos años la oferta de formación de grado y posgrado al respecto en el territorio nacional, con iniciativas variadas. En el caso de la Universidad de Málaga, hemos apostado por la enseñanza de grado con intensificación en Corea, desde una perspectiva transversal que recoge un amplio programa de estrategias de internacionalización at home y movilidad internacional de docentes y estudiantes, entre universidades de los dos países, sin olvidar una fuerte vocación de diálogo ibero-latinoamericano.

La puesta en marcha del nuevo título de grado representó un salto cualitativo fundamental en la configuración de un espacio propio de colaboración e investigación para el desarrollo de los estudios coreanos en el ámbito hispanoparlante. Hemos querido vertebrar este esfuerzo en torno a los siguientes ejes: transdisciplinariedad, interculturalidad y triangulación Latinoamérica-España-Corea. 


\section{Estudos da Ásia Oriental no âmbito hispanoparalante: A aposta da Universidade de Málaga}

Sumário

O presente artigo parte da idéia geral de que o conceito clássico de educação é profundamente afetado pela globalização contemporânea. Uma das expressões mais importantes da globalização no âmbito educativo é a internacionalização, principalmente na etapa de formação no nível superior. É assim que as universidades de todo o mundo consideram a internacionalização como um dos elementos privilegiados nos seus planos de desenvolvimento e de futuro.

Em esse contexto é que nas universidades da Espanha cresceu o interesse por intensificar a internacionalização com as universidades da Ásia Oriental, principalmente com as da China, o Japão e a Coréia do Sul. De igual modo, nos últimos anos se tem aumentado e diversificado, no território nacional e com iniciativas variadas, a oferta ao respeito do tema nos estudos de graduação e pós-graduação. No caso da Universidade de Málaga, a graduação apostou pelo ensino intensificado da Coréia desde uma perspectiva transversal que colhetou um grande programa de estratégias de internacionalização at home, e um programa de mobilidade internacional de professores e alunos entre as universidades dos dois países, sem se esqueçer da grande vocação do diálogo ibero-latinoamericano.

A implementação do novo título de graduação representou um salto qualitativo fundamental na configuração dum espaço próprio de colaboração e pesquisa para o desenvolvimento dos estudos coreanos no âmbito hispanoparlante. Se teve a intenção de articular esse esforço em redor dos seguientes eixos: transdisciplinariedade, interculturalidade e triangulação América Latina-Espanha-Coréia.

Palavras-chave

Globalização; Internacionalização da educação; Estudos da Ásia Oriental; Coréia do Sul.

\section{East Asian Studies in the Spanish-speaking sphere: The University of Malaga case}

\footnotetext{
Abstract

This research is based on the idea that the classic concept of education is profoundly affected by the contemporary globalisation. One of the most important expressions of globalisation in the educative field is internationalisation, especially in the university stage. Thus, higher education colleges from around the world consider internasionalisation as a privileged element in their development and future plans.

Spanish universities have a growing interest in this context, particularly, in strengthen internationalisation with East Asian universities, China, Japan and South Korea above all. In the same way, the offer of bachelor de- 
gree and postgraduate degree education has been widened and diversified in the last years.

In the University of Malaga, we have ventured into the bachelor degree teaching with intensification in Korea, from a transverse perspective that takes a wide program of "at home" internationalisation strategies, and international mobility of teachers and students between colleges of both countries, and with a strong vocation of a Ibero-Latino America dialogue.

The launch of the new degree represented an essential qualitative step to the configuration of an own space of collaboration and research, destined to the Korean studies development in the Spanish-speaking sphere. We have wanted to support this effort around three axes: transdisciplinarity, interculturalism and Latino America-Spain-Korea triangulation.

\section{Key words}

Globalisation; Internationalisation of higher education; East Asian studies; South Korea.

\section{Globalización y educación superior}

La globalización es un concepto polisémico hoy en día, que pretende designar un fenómeno histórico multidimensional de amplias resonancias, consecuencias y recorrido. En principio, se ha querido primar su carácter eminentemente económico $\mathrm{y}$ comercial, pero el avance tecnológico que ha acompañado, y vehiculado, a la globalización, así como el flujo consecuente de personas, ideas, artefactos varios, bienes y servicios, permite vislumbrar un proceso más amplio y diverso.

En realidad, la globalización tiene una historia más larga, que puede extenderse desde los inicios de la apertura de las rutas del Pacífico o en el siglo XVI hasta la actualidad, y que solo puede comprenderse en su historicidad, y no como expresión descontextualizada. Así, lo que Hausberger (2018) llama "globalización temprana" ya implicó a extensas regiones, como el este de Asia y México, y otros países latinoamericanos y España, como puerta para Europa, sin olvidar el papel del comercio portugués. Es en la globalización reciente que se condensan procesos de aceleración, solo intuidos hasta fechas recientes, con la regeneración continua de las TIC, que afectan intensamente las relaciones humanas y todo tipo de interacción económica, política, social, cultural, medioambiental, etc. (Rosenau, 2003).

También la educación está directamente implicada en estas transformaciones. Tiene un papel importante que jugar en estos procesos, pues, aunque el concepto clásico de educación se ve profundamente afectado por la globalización, la educación se convierte en puente necesario para atravesar estas nuevas fronteras, y herramienta esencial para afrontar los retos del nuevo orden global, más y nuevos conocimientos y habilidades, innovación, soluciones alternativas, etc. (Garratt, Phillips and Piper, 2003). 
Una de las más importantes expresiones de la globalización en el ámbito educativo es la internacionalización, sobre todo en la etapa de la formación universitaria. Las universidades de todo el mundo consideran la internacionalización como un elemento privilegiado en sus planes de desarrollo y futuro. Las estrategias de internacionalización contribuyen a aumentar la visibilidad de las universidades en un marco competitivo para lograr mayor relevancia académica. La oportuna labor de internacionalización facilita también el establecimiento de redes plurinacionales de investigación y cooperación, así como la llegada de inversiones, y el incremento del número de estudiantes. De igual modo, las posibilidades de intercambio y movilidad académica de estudiantes, profesores y empleados, suelen relacionarse con la mejora de competencias globales respecto a la empleabilidad y adaptabilidad a escenarios de naturaleza multicultural.

\section{Movilidad académica}

La movilidad académica no es exclusivamente una tendencia al alza entre las universidades de países occidentales. El número de estudiantes y profesores del área de Asia Oriental que participan en programas de educación superior en el extranjero ha crecido exponencialmente. Así, por ejemplo, algunos países que han asumido la globalización como una verdadera cuestión de Estado, como la República de Corea (Corea a partir de ahora en este texto), realizan importantes esfuerzos económicos, académicos y logísticos para ofrecer una gran variedad de opciones para enviar estudiantes al extranjero y atraer estudiantes foráneos (Kwon, 2013). En este sentido, están cambiando incluso las tendencias tradicionales de movilidad de países asiáticos hacia el ámbito anglosajón, y se buscan nuevos socios de intercambio, como es el caso de España, fundamentalmente considerada puente entre Europa y Latinoamérica.

A pesar de que se ha producido un retroceso consecutivo en el número total de estudiantes universitarios coreanos que realizan una estancia formativa en el extranjero entre 2012-2014, el país sigue representando uno de los puntos de origen más importantes para la movilidad académica internacional. Un informe de McKinsey \& Company en 2013 achaca este descenso a la propia disminución de la tasa de natalidad y a los efectos económicos sobre la clase media del adelgazamiento de los cuadros intermedios de muchas corporaciones (Choi, Dobbs, Suh, Mischke, Chon, Cho, Kim, and Kim, 2013). Así, después de varios años en el top 3 de los países de origen de la movilidad estudiantil, solo por detrás de China e India, en 2013 cedió esta posición a Alemania. En concreto, los principales países de destino de los estudiantes coreanos son, en este orden, Estados Unidos $(63,952)$, Japón $(14,958)$, Australia $(6,234)$, Gran Bretaña $(4,752)$ y Canadá $(4,685)$ (UNESCO, 2013). En los últimos años, se abren también otros destinos, fundamentalmente en el ámbito geoeconómico circundante y área del pacífico, como Nueva Zelanda y China-Hong Kong.

Por otra parte, la llegada de estudiantes internacionales a instituciones coreanas de enseñanza superior se ha elevado regularmente. El crecimiento total alcanzó el $7.6 \%$ en 2015 y el $14.2 \%$ en 2016 , con un número total de 104,262 estudiantes. Estos datos recogen el aumento progresivo de estu- 
diantes de Asia Central, del Sur, y Sureste, aunque esta progresión quede ensombrecida por sentimientos de discriminación y peor recibimiento y trato en comparación con estudiantes de otros países, según reflejan algunas investigaciones recientes (World Education Services, 2017). En concreto los países de origen mayoritarios de estos desplazamientos hacia universidades coreanas son China $(34,513)$, Vietnam $(2,548)$, Mongolia $(2,129)$, Estados Unidos $(1,355)$, Taiwán $(1,286)$ e Indonesia (841) (UNESCO, 2013).

Por su parte, el sistema universitario español afirma estar implicado en una estrategia de internacionalización de los programas educativos, ofreciendo oportunidades de aprender y adquirir competencias para trabajar en entornos transnacionales, como habilidades lingüísticas y comunicativas, interculturales, habilidades para el trabajo en grupo, emprendimiento, liderazgo, entre otras (MECD, 2016). Sin embargo, podemos detectar algunos problemas para alcanzar un objetivo tan ambicioso, por ejemplo, el número inferior de estudiantes internacionales que deciden realizar sus estudios universitarios, de grado, máster o doctorado, en universidades españolas respecto a los datos de otros países desarrollados. Así, solo el $2.8 \%$ de los estudiantes en el sistema español eligen España para cursar sus estudios de grado o posgrado (ajenos a la movilidad temporal de uno o dos semestres), en contraste con otros países de su entorno, como el 4\% en Italia o el 4.7 en Portugal (Michavila, Martínez \& Merhi, 2015). Sin embargo, la universidad española es el principal emisor y receptor de estudiantes de movilidad temporal en Europa, consiguiéndose un balance positivo en los últimos años para la salida de estudiantes hacia el extranjero.

Precisamente, en 2017 se han cumplido treinta años del famoso programa de movilidad Erasmus, denominado en la actualidad Erasmus+, cuya aplicación se extenderá hasta 2020. En la convocatoria de 2014, 42,537 estudiantes de Educación Superior eligieron España para cursar su Erasmus, por delante de Alemania $(32,871)$, Reino Unido $(30,183)$, Francia $(29,558)$ e Italia $(21,564)$. España ocupa esta primera posición de manera ininterrumpida desde el año 2001. En estos 30 años del Programa Erasmus, España ha pasado de enviar 95 alumnos (año 1987) a casi 42,000 participantes (en 2014). Asimismo, se ha incrementado un $14.86 \%$ el número de estudiantes que realizó prácticas en empresas, lo que sitúa a España en el grupo de cabeza en este tipo de movilidad, por detrás solo de Alemania y Francia. Entre las razones para este liderazgo se esgrime la amplia oferta formativa, el interés por la lengua y la cultura, el estilo de vida, el clima y el gran atractivo turístico, condiciones que también son valoradas por los participantes no europeos de este tipo de intercambios (Pérez-Encinas, Howard, Rubley y De Witt, 2017). 


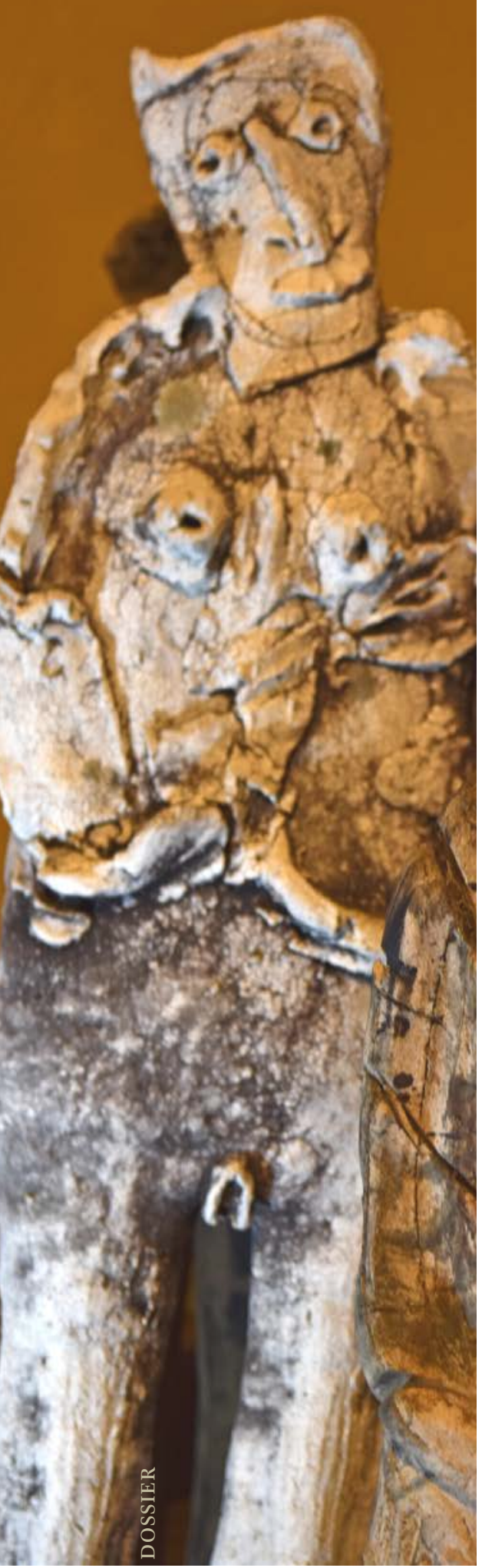

\section{El contexto español: estudios asiáticos en la Universidad de Málaga}

Es en este contexto que entre las universidades españolas crece el interés por intensificar la internacionalización con universidades de Asia Oriental, sobre todo China, Japón y Corea, generándose una actividad intensa y cierta competitividad por establecer lazos y acuerdos interuniversitarios (Del Águila, Montalbán y Padilla, 2017). Los intercambios académicos entre Corea y España se centran en movilidades recíprocas ligadas sobre todo a la enseñanza del idioma y a programas específicos dentro de convenios de colaboración interuniversitarios. Para España, Corea representa un socio minoritario todavía, pero emergente, en la estrategia de internacionalización, definiendo las autoridades españolas la educación en el país asiático como de calidad y orientada a las nuevas tecnologías. Concretamente, la movilidad correspondió en 2013 a 32 estudiantes de salida desde España y 81 estudiantes coreanos recibidos en universidades españolas (UNESCO, 2013). En general, para este tipo de movilidad se recurre a programas co-financiados por la Unión Europea y los países socios, que apoyan el intercambio de estudiantes y personal académico asociado a proyectos de colaboración en educación e investigación, promoviendo la internacionalización de las universidades y personal académico, incluyendo la posibilidad para los jóvenes de adquirir experiencia laboral o profesional temporal. Recientemente también, el Consejo de Ministros del gobierno español (1 de diciembre de 2017) ha autorizado la firma de un acuerdo con el gobierno de la República de Corea relativo a un programa de movilidad juvenil con el que se beneficiarán jóvenes de 18 a 30 años de ambos países, que podrán compaginar las vacaciones con la adquisición de conocimientos de la cultura del otro país (Gobierno de España, 2017).

La Universidad de Málaga (UMA, a partir de ahora) trabaja desde 2006 para convertirse en socio prioritario en el ámbito iberoamericano de universidades e instituciones coreanas. En 2010 este proyecto se concreta en un acuerdo con la Universidad Nacional de Incheon para construir canales fluidos de colaboración en proyectos

46 Universidades $\mid$ núm. 84, abril-junio 2020 UDUAL | DOI:https://doi.org/10.36888/udual.universidades.2020.84.103

Manuel Montalbán y María Auxiliadora Durán | Asimetrías y consolidación progresiva de los intercambios académicos entre México y Japón, siglo XXI 
académicos, de investigación, de emprendimiento empresarial, etc. La mayoría de estas iniciativas se recogen en el marco del Campus de Excelencia Internacional Andalucía-TECH, que conformaron las Universidades de Sevilla y Málaga. Este nuevo espacio de intercambio permitió gestar una amplia colaboración entre ambas instituciones, orientada al desarrollo de actuaciones en el ámbito de la investigación y la transferencia de conocimiento, apostando ambas instituciones por la excelencia docente e investigadora, la internacionalización y la captación de talento. Ese espíritu común animó, entre otros objetivos, el desarrollo de seis titulaciones de grados conjuntas, junto a otras de especial interés para la especialización del campus de excelencia.

Si bien la mayor parte de su oferta formativa estuvo orientada al ámbito de carreras técnicas (Ingeniería de la Energía o Ingeniería en Electrónica, Robótica y Mercatrónica), tuvo cabida la propuesta de un título conjunto en Estudios de Asia Oriental que comienza su andadura en el curso 2011-2012. Este nuevo grado fue propuesto con una estructura que reconoce tres menciones de especialización para el alumnado: China, Japón y Corea. Las menciones están distribuidas en cuanto a docencia entre ambas sedes: China y Japón pueden cursarse en Sevilla, quedando emplazada en la UMA la mención Corea como colofón a las distintas iniciativas académicas y culturales precedentes. Esta mención aspira a capacitar a profesionales como intermediarios culturales entre el mundo de habla española y el mundo de los negocios, la cultura, las relaciones internacionales, etc. en Corea (Doménech, Montalbán, Llorente, Zurita, Kang, Botella \& Chica, 2016).

Se ponía así de manifiesto el fuerte vínculo de la UMA con universidades coreanas, en especial con la Universidad Nacional de Incheon. Reflejo de este trabajo conjunto, en el año 2019 se ha celebrado el $\mathrm{x}$ Aniversario del inicio de esta apuesta común, uno de cuyos símbolos más destacados es la presencia de la Oficina Puente con Corea en la UMA, y de su equivalente por parte de la Universidad de Málaga en Incheon. La labor de ambas entidades está orientada a favorecer los intercambios académicos y culturales, desarrollando una labor de enlace entre ambos

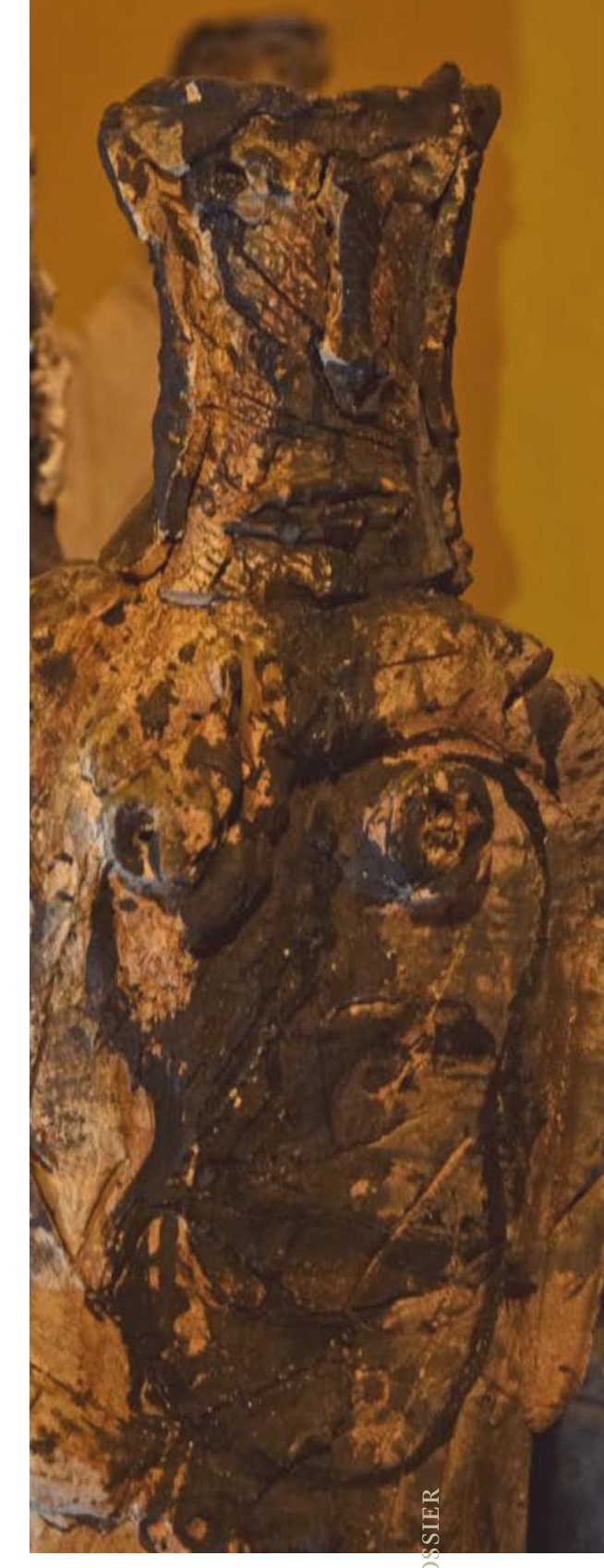


países. En esta línea de difusión, y fruto del interés por lo intercultural, anualmente se ha venido celebrando la Semana de Corea, cuyo programa de actividades abarca tanto el contexto universitario como diversos espacios culturales de la ciudad. Estas iniciativas han aumentado el número de intercambios académicos UMA-Corea, sobre todo por parte de los estudiantes coreanos. De forma recurrente en estos años el número de estudiantes procedentes de y con destino a la Universidad Nacional de Incheon es el más elevado en los intercambios de movilidad que se producen entre la UMA y diversas universidades coreanas.

Centrándonos en la formación en Estudios de Asia Oriental, el Grado se ofertó por primera vez en el curso académico 2011-12, habiendo egresado en la actualidad cinco promociones. El alumnado a lo largo de estos años se ha mantenido estable en cuanto a la demanda de plazas, y suelen cubrirse todas las de nuevo ingreso en primero (en torno a 50 plazas anuales en el marco de la oferta de Distrito único de las universidades andaluzas).

La estructura del título recoge materias de formación básica, obligatorias, optativas de mención (aunque en la práctica funcionan como asignaturas obligatorias debido a la oferta de créditos a cursar) y optativas. En esta configuración es requisito para el alumnado cursar un itinerario específico, el cual incluye las asignaturas de idioma, junto a las obligatorias de mención y optativas correspondientes, para obtener cada una de las menciones. El total de créditos a cursar es de 240, quedando distribuidos del siguiente modo:

Tabla 1. Distribución de créditos ECTS del Plan de Estudios del Grado en Estudios de Asia Oriental según el tipo de materia

\begin{tabular}{|c|c|}
\hline Tipo de materia & Créditos \\
\hline Formación Básica & 60 \\
\hline Obligatorias & 48 \\
\hline Optativas & 126 \\
\hline Trabajo Fin de Grado & 6 \\
\hline Total & 240 \\
\hline
\end{tabular}

Fuente: Resolución 8230 BOE (jueves 13 de julio de 2017, número 166; Sec. III. p. 61516).

En la Tabla 2 se recogen, como ejemplo de un itinerario dentro del Grado conjunto, las diferentes asignaturas de la titulación estructuradas por módulos tras la modificación del plan de estudios de Graduado en Estudios de Asia Oriental según la Resolución del 31 de mayo de 2017 (Resolución 8230 BOE, jueves 13 de julio de 2017, número 166; Sec. III). 
Tabla 2. Módulos y asignaturas del Grado en Estudios de Asia Oriental (Universidad de Sevilla/ Universidad de Málaga)

\begin{tabular}{|c|c|c|c|}
\hline Módulo & Asignatura & Carácter & Créditos \\
\hline \multirow{10}{*}{$\begin{array}{l}\text { Formación } \\
\text { Básica }\end{array}$} & Arte y Estética en Asia Oriental & $\mathrm{F}$ & 6 \\
\hline & Comercio y Relaciones Internacionales de Asia Oriental & $\mathrm{F}$ & 6 \\
\hline & Economía Contemporánea de Asia Oriental & $\mathrm{F}$ & 6 \\
\hline & Geografía de Asia & $\mathrm{F}$ & 6 \\
\hline & Historia de las Religiones de Asia Oriental & $\mathrm{F}$ & 6 \\
\hline & Historia Premoderna de Asia Oriental & $\mathrm{F}$ & 6 \\
\hline & Literaturas Clásicas de Asia Oriental & $\mathrm{F}$ & 6 \\
\hline & Mediación y Negociación Intercultural con Asia Oriental & $\mathrm{F}$ & 6 \\
\hline & Modelos de Pensamiento en Oriente y Occidente & $\mathrm{F}$ & 6 \\
\hline & Sistemas Políticos Contemporáneos de Asia Oriental & $\mathrm{F}$ & 6 \\
\hline \multirow{8}{*}{ Idiomas } & Coreano I (CO) & $P$ & 6 \\
\hline & Coreano II (CO) & $P$ & 6 \\
\hline & Coreano III (CO) & $P$ & 6 \\
\hline & Coreano IV (CO) & $P$ & 6 \\
\hline & Coreano V (CO) & $P$ & 6 \\
\hline & Coreano VI (CO) & $P$ & 6 \\
\hline & Coreano VII (CO) & $P$ & 6 \\
\hline & Coreano VIII (CO) & $P$ & 6 \\
\hline \multirow{10}{*}{ Optativas } & Escénicas, Danza y Música en Corea (CO) & $P$ & 6 \\
\hline & Chamanismo, Naturaleza y Prácticas Religiosas (CO) & $P$ & 6 \\
\hline & Coreano para los Negocios (CO). & $P$ & 6 \\
\hline & Cultura Empresarial y Negociación en Corea (CO) & $P$ & 6 \\
\hline & Cultura Popular y Contemporánea en Corea (CO) & $P$ & 6 \\
\hline & Marcos de Innovación y Nuevas Tecnologías (CO) & $P$ & 6 \\
\hline & Medicina Tradicional y Salud en Asia Oriental $(\mathrm{JA}, \mathrm{CH}, \mathrm{CO})$ & $P$ & 6 \\
\hline & Relaciones de España y Latinoamérica con Corea (CO) & $P$ & 6 \\
\hline & Turismo e Intercambios Culturales y Educativos con Corea (CO) & $P$ & 6 \\
\hline & Prácticas Externas & $\mathrm{P}$ & 18 \\
\hline \multirow{13}{*}{$\begin{array}{l}\text { Sociedades } \\
\text { y culturas }\end{array}$} & Economía de las Empresas Asiáticas y su penetración en España & & \\
\hline & e lberoamérica & 0 & 6 \\
\hline & Historia Contemporánea de Asia Oriental & $\mathrm{O}$ & 6 \\
\hline & Cultura Digital y Nuevas Tecnologías de la Información y la & $\mathrm{O}$ & 6 \\
\hline & Comunicación en Asia Oriental & $\mathrm{O}$ & 6 \\
\hline & Economía y Finanzas de Asia Oriental & $\mathrm{O}$ & 6 \\
\hline & Escrituras Contemporáneas de Asia Oriental & $\mathrm{P}$ & 6 \\
\hline & Estética y Arte Coreanos (CO) & O & 6 \\
\hline & Historia Moderna de Asia Oriental & $P$ & 6 \\
\hline & Literatura de Corea a Través de sus Textos (CO) & $P$ & 6 \\
\hline & Pensamiento y Religiones en Corea a Través de sus Textos (CO) & $P$ & 6 \\
\hline & Política y Geoestrategia en Corea (CO) & O & 6 \\
\hline & $\begin{array}{l}\text { Teorías del Diálogo y la Interculturalidad } \\
\text { Turismo y Flujos Migratorios en Asia Oriental }\end{array}$ & O & 6 \\
\hline $\begin{array}{l}\text { Trabajo Fin } \\
\text { de Grado }\end{array}$ & Trabajo Fin de Grado & $\mathrm{T}$ & 6 \\
\hline
\end{tabular}

Fuente: Adaptado de Resolución 8230, BOE (jueves 13 de julio de 2017, número 166) // Nota: F (Formación Básica); P (Optativas); O (Obligatorias); T (Trabajo Fin Grado) // CH: China; JA: Japón; CO: Corea. 
Esta configuración de materias se orienta a lograr que el alumnado alcance competencias generales en el ámbito de los Estudios de Asia Oriental, al tiempo que permite focalizar el objeto de estudio en China/ Japón/Corea a través del aprendizaje del idioma y del estudio de aspectos culturales, sociales, o económicos más específicos. Esa dualidad hace posible diferenciar la titulación de otras ofertas formativas que enfatizan la competencia lingüística o consideran algunas de estas menciones como una formación complementaria (estudiando el idioma coreano como segunda opción, o incluyendo algunas optativas vinculadas a Corea en la oferta general).

\section{Enfocando los estudios coreanos desde el mundo hispanoparlante}

En definitiva, la puesta en marcha del nuevo título de grado representó un salto cualitativo fundamental en la configuración de un espacio propio de colaboración e investigación para el desarrollo de los estudios coreanos en el ámbito hispanoparlante. Podemos vertebrar este esfuerzo en torno a los siguientes ejes.

\section{Transdisciplinariedad}

La puesta en marcha del Espacio Común Europeo de Enseñanza Superior conllevó una importante transformación de la oferta de los estudios universitarios tanto en su diseño formal y de nuevos contenidos metodológicos. Se conformaron redes interuniversitarias de reflexión sobre el estado del arte en cada disciplina y desde la administración educativa se animó la realización de Libros Blancos. En el caso de los Estudios de Asia Oriental existía ya alguna experiencia previa plasmada en la oferta de licenciaturas de segundo ciclo a las que se podía acceder desde estudios universitarios previos. Sin embargo, esta andadura no cristalizó en ningún Libro Blanco específico, sino que se asimiló al área de la enseñanza de las lenguas y sus culturas, desde la que, se apostaba por una orientación inclusiva de disciplinas que ayudaran a comprender el hecho diferencial nacional, y regional, y su inserción en un mundo globalizado, más allá de las materias de índole lingüística y literaria (Elizalde, 2007). 
Así, los Estudios de Asia Oriental en España, organizados ya bajo la fórmula de grado y master, reflejan cuando menos una aproximación multidisciplinar con temáticas, además de naturaleza idiomática, relativas a historia, geografía, antropología, sociedad, arte y cultura, economía, comercio, ciencia política, relaciones internacionales, negocios, etc. Desde la puesta en marcha de la titulación de la UMA se han sucedido diferentes iniciativas de coordinación para conseguir alcanzar un paso más en la mera diversidad temática y diseñar iniciativas transdisciplinares, sobre todo a través de proyectos de innovación educativa y de investigación que afronten retos formativos y objetos de estudio de manera conjunta.

\section{Interculturalidad}

En el título, considerándolo como ilustración privilegiada de internationalization at home, y siendo conscientes del papel fundamental que la movilidad académica, altamente incentivada en los estudios, tiene para nuestros alumnos, hemos apostado por una concepción avanzada de competencia intercultural que abarque actitudes, habilidades y conocimientos, que permitan evaluar, y responder con eficacia, a los requerimientos interpersonales, emocionales, educativos, organizacionales, derivados de la diversidad cultural. Queremos tener presente la recomendación de Dervin (2011) acerca de la idea extendida de enriquecimiento abstracto que supone cualquier experiencia de movilidad. El autor insta pues a transcender los discursos contradictorios y circulares sobre las relaciones entre movilidad académica, internacionalización e interculturalidad. Lo intercultural representa un desafío que apunta a transformar las ideas preconcebidas y las opiniones poco contrastadas acerca del otro. Se trata de un desafío para transcender el culturalismo rígido, que justifica la descripción multicultural, esencializando las diferencias culturales, y definiendo lo intercultural como viaje de ida y vuelta entre universos culturales cerrados.

De esta manera, se han desarrollado algunos proyectos empíricos para comprender mejor la experiencia de movilidad de nuestros estudiantes. Podemos destacar el proyecto de investigación "Building the Future: Korean Studies Postgraduate Program and Research Development at the University of Malaga", financiado por The Academy of Korean Studies durante el periodo 2016-2019 (Seed Program for Korean Studies), que ha puesto de manifiesto la existencia de toda una serie de condiciones que determinan un aprovechamiento de estos desplazamientos académicos desde el punto de vista intercultural: nivel de conocimiento de la lengua en el país de destino, motivación para la estancia, búsqueda y apertura a contactos en destino, relaciones interpersonales derivadas, etc.

Recientemente se ha realizado también desde la UMA un estudio sobre la presencia de competencias interculturales en los planes existentes corroborando que en los casos analizados se prima el conocimiento sociolingüístico e instrumental, y el título conjunto de la Universidades de Sevilla-Málaga-Campus Andalucía TECH destaca por un perfil más centrado en las competencias interculturales (Del Águila, Montalbán y Padilla, 2017). 


\section{Triangulación España-Latinoamérica-Corea}

También al comienzo de la década del 2000 el interés internacional que desde España se mostraba hacia las emergentes economías del este asiático se reforzaba con la hipótesis de que el país ibérico pudiera asumir el papel de puente entre China, Japón y Corea, y los países latinoamericanos, fundamentado además en su inclusión en el marco común de la Unión Europea. Esta apuesta, más retórica que pragmática, ha mostrado diversas intensidades en sus formas de presentación a lo largo de estos años, dependiendo de los ciclos políticos y económicos, internos y en la cooperación iberoamericana, y en la actualidad necesita ser redefinida, sobre todo a partir de la constancia de datos macroeconómicos, y diplomáticos, que corroboran la potencia de alianzas regionales Asia-Pacífico, así como la relevancia de las relaciones bilaterales directas de los países latinoamericanos y de Asia Oriental.

Aun así el título ha optado por explorar estas opciones, incluyendo materias específicas, pero sobre todo a través del establecimiento de contactos e incorporación a redes interuniversitarias con instituciones latinoamericanas con oferta o intereses en los estudios asiáticos. Los intercambios y colaboraciones, en este sentido, han ido creciendo exponencialmente. Existen, por ejemplo, acuerdos triangulares que incluyen a la Incheon $\mathrm{Na}$ tional University y a la Universidad Autónoma de Nuevo León. La UMA está integrada igualmente en el consorcio de universidades del eSchool Program for Latin America de la Korea Foundation. La presencia de investigadores de la UMA también es habitual en encuentros y foros en tierras latinoamericanas, ALADAA, WCAAS, EECAL, etc. Asimismo, desde la UMA se han organizado tres encuentros iberoamericanos de estudios coreanos bianuales, que han reunido a expertos de Corea, Latinoamérica y España, en torno a aspectos como colaboración y movilidad académica, interculturalidad, juventud y cambios sociales, etc.

De estas iniciativas se deriva la necesidad de redefinir el concepto de triangulación desde una perspectiva académica, pues es en esta dimensión que ofrece grandes posibilidades de construcción de un espacio común para enriquecer con perspectiva de futuro los estudios coreanos, no solo desde una perspectiva regional, histórica o de similitudes culturales entre España y Latinoamérica. Las sinergias despertadas evidencian la necesidad de combinar y compensar fortalezas y debilidades. España presenta un mapa académico en proceso de consolidación en la oferta de Estudios de Asia Oriental, y en particular en los estudios coreanos, por lo que a la UMA afecta. No obstante, la oferta continuada de los últimos años obliga también a explorar nuevos horizontes de inserción e influencia de la masa crítica generada, sobre todo teniendo en cuenta que las relaciones económicas, comerciales y culturales de España con Corea, aunque reflejan buenas expectativas, son poco significativas si las comparamos con las de algunos países latinoamericanos como Chile, Perú, Colombia o México. En Latinoamérica, sin embargo, y a pesar de las contrastadas tradiciones existentes en algunos países enraizadas en la historia de relaciones bilaterales y en la existencia de diáspora con flujos diversos, la oferta de educación superior formal especializada es bastante limitada. 


\section{Retos comunes en el ámbito de los estudios coreanos}

Prestando atención a la reciente implantación de los Estudios de Asia Oriental en las Universidades de Sevilla y Málaga son aún muchos los retos propios planteados. Entre otros hemos destacado como apuestas claras para su consolidación el desarrollo de propuestas de formación de posgrado específicas, avanzar en la identificación de trayectorias de empleabilidad del alumnado egresado y los vínculos con los potenciales empleadores, o la puesta en marcha de equipos multidisciplinares transnacionales que permitan afianzar líneas de investigación en este ámbito y fomenten la transmisión del conocimiento a la sociedad.

Para completar nuestro recorrido incluimos una mirada a otras latitudes. Así algunos de los retos que recoge el informe Future Visions. Opportunities and Challenges of Korean Studies in North America (2018), fruto de las jornadas organizadas por el Korea Program-Shorenstein APARC y cuyas conclusiones ha publicado la Universidad de Stanford, pueden ser generalizados a los estudios coreanos en España y al status quo de los estudios coreanos en general. Salvando la distancia que supone la ampliamente consolidada tradición de este ámbito en Estados Unidos, podemos asumir como propio el reto de desarrollar una narrativa compartida, interdisciplinar, con el fin de afirmar la relevancia de este ámbito de estudio para un público más amplio, siendo así que el futuro de los estudios coreanos descansaría en la transformación del apasionado y extendido interés en la cultura pop coreana en un interés académico duradero. Este interés suscitado por el nuevo contexto coreano ha contribuido a que la matriculación en cursos de coreano se haya incrementado notablemente en un entorno como el estadounidense, de reducido interés en el aprendizaje de lenguas extranjeras, y destaca el hecho de que muchos de estos estudiantes no están vinculados a Corea por lazos familiares. Se nos ofrece así una figura similar a la encontrada en la Universidad de Málaga; sin embargo, este prometedor inicio se ve comprometido debido, en parte, al limitado número de horas de idiomas incluidas en los programas formativos de grado y a la ausencia de posgrados específicos, tanto vinculados al estudio de la lengua como de otras disciplinas. 
Otro importante punto de confluencia con nuestro propio desarrollo como titulación es el inestimable apoyo continuado recibido de instituciones como la Korea Foundation. Junto a esta valoración positiva, podría apuntarse como una debilidad de la formación ofrecida la dependencia que conlleva, en especial para el aprendizaje del idioma, siendo necesario que esa inversión se mantenga a largo plazo y pueda traducirse en oportunidades para los estudiantes tanto de incrementar su nivel de coreano como de vivir una experiencia de inmersión cultural en Corea durante un período prolongado.

Desde una perspectiva más académica podríamos sumarnos a uno de los retos esenciales planteados en estas jornadas: propiciar un giro copernicano que posibilitara a los estudios coreanos avanzar desde un enfoque centrado en "qué podemos aprender sobre Corea" a otro que nos permita orientarnos a "qué podemos aprender de Corea" (y por qué no: "qué podemos aprender con Corea") dando carta de naturaleza a la doble vía en los procesos de globalización. En este sentido, una aportación fundamental que pueden llevar adelante los coreanistas, en sus respectivas disciplinas de origen, se vincula a la puesta en cuestión tanto del eurocentrismo como del sinocentrismo, funcionando como antídoto frente a un entorno académico que toma la experiencia occidental como dogma, o tiende a asimilar China como equivalente de Asia Oriental en conjunto. La comunicación fluida con académicos coreanos y la estructuración de líneas de investigación conjuntas apoyaría el desarrollo de una comunidad académica crítica global en este ámbito de estudio.

A pesar de que en el ámbito de las ciencias sociales, Corea se valora como un entorno cuasi-experimental, reflejo de una modernidad condensada, la inercia académica y las tendencias de las principales publicaciones disciplinares suponen aún un cierto obstáculo al desarrollo de investigación autóctona en el marco coreano. La presión a colocar publicaciones en revistas de impacto también afecta este avance, pues resulta complejo desde este ámbito publicar en revistas destacadas de la propia disciplina de origen (a excepción del ámbito de gestión de empresas), manteniéndose la tendencia al crecimiento de las publicaciones en revistas consideradas de estudios de área.

En este sentido, consideramos que se hace necesario un diálogo mas intenso entre investigadores y académicos de ambos lados del Atlántico, lo que puede desembocar en la actualización de las agendas empíricas y de debate sobre los estudios coreanos en los países hispanoparlantes. Temas sobre responsabilidad social, desarrollo humano y territorial, flujos migratorios, interculturalidad, incluso comparativas de afrontamiento de retos sociales en Corea y Latinoamérica, pueden sumarse a otros con más larga presencia, e ilustrar que la globalización no es únicamente un viaje de ida. 


\section{Referencias}

Del Águila, A.R., Montalbán, F.M., y Padilla, M. (2017). Asian studies degrees and intercultural competence: The case of Spain, KEDI Journal of Educational Policy, 14:1, 45-58.

Choi, W., Dobbs, R., Suh, D., Mischke, J., Chon, E., Cho, H., Kim, B., y Kim, H. (2013). Beyond Korean style: Shaping a new growth formula. McKinsey Global Institute, April. Disponible en https:// www.mckinsey.com/global-themes/asia-pacific/beyond-korean-style

Dervin, F. (2011). Introduction, En F. Dervin (Ed.) Analysing the consequences of academic mobility and migration. Cambridge, U.K.: Cambridge Scholars Publishing.

Doménech, A.J., Montalbán, F.M., Llorente, F.M., Zurita, E., Kang, E.K., Botella, L.A., y Chica, P. (2016). We-Uri 2.0: Identities and Transcultural Mediations in Korea-Spain Academic Exchanges, 8th World Congress for Korean Studies, 5-7 october, Center for East Asian Studies, University of Pennsylvania disponible en http://congress.aks.ac.kr/korean/files/2_1478847096.pdf

Elizalde, M. D. (2007) .La investigación sobre Asia y el Pacífico en España en el área de las ciencias humanas y sociales, Anuario Asia Pacífico 2006. Barcelona: CIDOB, Casa Asia y Real Instituto Elcano, 495-506.

Future Visions. Opportunities and Challenges of Korean Studies in North America. Conference report December 2018 [en línea]. Stanford-Korea Program. Shorenstein APARC // Foundation Academia Platonica. Disponible en: https://fsi.stanford.edu/publication/future-visions-opportunities-and-challenges-korean-studies-north-america

Garratt, D., Phillips, R. and Piper, H. (2003). Globalisation and its Impact Upon Education: prospects and challenges. British Educational Research Journal, 29: 441-449. doi:10.1080/01411920301840

Gobierno de España (2017). ACUERDO por el que se autoriza la firma del Acuerdo entre el Gobierno del Reino de España y el Gobierno de la República de Corea relativo al Programa de movilidad juvenil. Disponible enhttp://www.lamoncloa.gob.es/consejodeministros/referencias/Paginas/2017/refc20171201.aspx

Hausberger, B. (2017). Historia mínima de la globalización temprana. Ciudad de México: El Colegio de México.

Kwon, K-S (2013). Government Policy and Internationalization of Universities: The Case of International Student Mobility in South Korea, Journal of Contemporary Eastern Asia, 12, 1, 35-47.

MECD (2016). Internationalization strategy. Spanish Ministry of Education. Disponible en http://www. mecd.gob.es/educacion-mecd/dms/mecd/educacion-mecd/areas-educacion/universidades/ politica-internacional/estrategia-internacionalizacion/EstrategiaInternacionalizaci-n-Final. pdf

Michavila, F., Martínez, J. M., \& Merhi, R. (2015). Comparación internacional del Sistema Universitario Español. CRUE Universidades Españolas. Disponible en http://www.crue.org/Documentos\%20compartidos/Publicaciones/Monograf\%C3\%ADas/Monografia_Web_Comparacion. pdf

Pérez-Encinas, A., Howard, L., Rubley, L., y De Witt, H. (2017). The internationalisation of higher education in Spain. Madrid: SEPIE.

Resolución 8230 de 31 de mayo de 2017, conjunta de la Universidad de Sevilla y de la Universidad de Málaga, por la que se publica la modificación del plan de estudios de Graduado en Estudios de Asia Oriental (Grado conjunto de las universidades de Sevilla y Málaga). Boletín oficial del Estado, 13 de julio de 2017, núm 166, pp. 61516-61520 [Consultado el 26 Octubre 2019] Disponible en: https://www.boe.es/boe/dias/2017/07/13/pdfs/BOE-A-2017-8230.pdf

Rosenau, J. (2003). Distant Proximities: Dynamics Beyond Globalisation. Princeton: Princeton University Press.

UNESCO (2013). Global Flow of Tertiary-Level Students. Disponible en http://uis.unesco.org/en/ uis-student-flow 